\title{
Unstable plaques hide in heavily calcified coronary arteries
}

\author{
Wei-Hua Yin ${ }^{1 \#}$, Hong-Yue Wang ${ }^{2 \#}$, Yang Sun ${ }^{2}$, Xiang-Nan $\mathrm{Li}^{1}$, Zhong-Fei Lu ${ }^{1}$, Yun-Qiang An ${ }^{1}$, \\ Zhi-Hui Hou ${ }^{1}$, Jie Zhang ${ }^{1}$, Xin-Shuang Ren ${ }^{1}$, U. Joseph Schoepf ${ }^{3}$, Yan Zhang ${ }^{1,4 *}$, Bin Lu ${ }^{1 *}$ \\ ${ }^{1}$ Department of Radiology, Fuwai Hospital, State Key Laboratory of Cardiovascular Disease, National Center for Cardiovascular Diseases, Chinese \\ Academy of Medical Sciences and Peking Union Medical College, Beijing, China; ${ }^{2}$ Department of Pathology, Fuwai Hospital, State Key Laboratory \\ of Cardiovascular Disease, National Center for Cardiovascular Diseases, Chinese Academy of Medical Sciences and Peking Union Medical College, \\ Beijing, China; ${ }^{3}$ Department of Radiology and Radiological Science, Medical University of South Carolina Heart \& Vascular Center, Charleston, \\ SC, USA; ${ }^{4}$ Department of Radiology, Affiliated Hospital of Guizhou Medical University, Guiyang, China
}

Contributions: (I) Conception and design: All authors; (II) Administrative support: XN Li, YQ An, ZH Hou, J Zhang, XS Ren; (III) Provision of study materials or patients: WH Yin, HY Wang, Y Sun; (IV) Collection and assembly of data: WH Yin, HY Wang, Y Sun, XN Li, YQ An, ZF Lu, ZH Hou, J Zhang, XS Ren, Y Zhang, B Lu; (V) Data analysis and interpretation: WH Yin, HY Wang, Y Sun, UJ Schoepf, XN Li, ZF Lu, ZH Hou, J Zhang, XS Ren, Y Zhang, B Lu; (VI) Manuscript writing: All authors; (VII) Final approval of manuscript: All authors.

"These authors contributed equally to this work.

*These authors contributed equally to this work.

Correspondence to: Dr. Bin Lu, MD. Department of Radiology, Fuwai Hospital, State Key Laboratory of Cardiovascular Disease, National Center for Cardiovascular Diseases, Chinese Academy of Medical Sciences and Peking Union Medical College, \#167 Bei-Li-Shi Street, Beijing 100037, China. Email: blu@vip.sina.com or lubinvip1@163.com; Dr. Yan Zhang, MD. Department of Radiology, Affiliated Hospital of Guizhou Medical University, Guiyang 550004, China. Email: zoe040900@live.cn.

Background: The napkin-ring sign (NRS) was accepted as unstable plaques at coronary computed tomography angiography (CCTA). However, the incidence is relatively low. We sought to assess whether the newly defined diamond-attenuation-sign [DAS, defined as a qualitative plaque feature in a mixed plaque (MP) on CCTA cross-section images by the presence of two features: a visual calcification (in the shape of a diamond) accompanied by an annular-shape lower attenuation plaque tissue surrounding the lumen like a ring], could be accurately identified as unstable atherosclerotic plaques.

Methods: Eight heart transplant recipients (8 male; mean age, 48.5 \pm 11.6 years; range, 37-65 years) underwent CCTA exams prior to heart transplant surgery. Segment-based CCTA sections were independently evaluated for various plaque patterns including non-calcified plaque (NCP) with NRS (NCPNRS), NCP without NRS (NCP-non-NRS), MP with DAS (MP-DAS), MP without DAS sign (MP-nonDAS), and calcified plaque (CP).

Results: NCP-NRS plaques in 6.4\% (23/358), NCP-non-NRS plaques in 24.0\% (86/358), MP-DAS plaques in $18.2 \%$ (65/358), MP-non-DAS plaques in 20.1\% (72/358), and calcified-plaques in 7.0\% (25/358) of all cases. The specificity and positive predictive values of the MP-DAS and NCP-NRS signs to identify unstable plaque features were excellent (97.1\% vs. 98.6\%, 90.8\% vs. 87.0\%, respectively). DAS plaques were more frequently seen on CCTA exams than that of NRS (39.3\% vs. $13.3 \%$, respectively, $\mathrm{P}=0.001)$. The diagnostic performance of MP-DAS to identify unstable coronary lesions was superior compared to NCPNRS [area under the receiver operating characteristic curve (ROC), 0.756; 95\% CI: 0.717-0.791 vs. 0.558 ; 95\% CI: 0.514-0.600, respectively, $\mathrm{P}<0.001]$.

Conclusions: Both the DAS and NRS had a high specificity and positive predictive value for the presence of unstable lesions. DAS was a better identification of unstable atherosclerotic plaques in the assessment of plaque-calcification-pattern (PCP). 
Keywords: Atherosclerosis; unstable plaque; computed tomography angiography; coronary artery disease

Submitted Jul 27, 2021. Accepted for publication Dec 31, 2021; Published online: 21 Feb 2022.

doi: 10.21037/qims-21-521

View this article at: https://dx.doi.org/10.21037/qims-21-521

\section{Introduction}

Cardiovascular diseases are projected to remain the leading cause of death by 2030 globally (1). The American Heart Association updated the classifications to define calcified plaques (CP), such as the type VII coronary plaque, which occur as part of the atherosclerotic process and are associated with a worse prognosis $(2,3)$. However, since stable and unstable plaques were defined in 1988, it has remained controversial whether atherosclerotic calcified lesions are stable or not.

Coronary computed tomography angiography (CCTA) is the main noninvasive tool for evaluation of coronary CPs $(4,5)$ and the differentiation between calcified and non-calcified plaque (NCP) components had already been described with early multi-detector CT technology (6). The napkin-ring sign (NRS) was accepted as an important NCP feature at CCTA to identify unstable plaques. However, the incidence of NRS is relatively low (7).

In this study, we sought to identify the diagnostic performance of a plaque-calcification-pattern (PCP) scheme based on CCTA to differentiate between stable and unstable atherosclerotic plaques as defined by histopathology. Moreover, we sought to assess whether the newly defined diamond-attenuation sign (DAS), a CT plaque feature in vivo, could be accurately identified as unstable atherosclerotic plaques. We present this article in accordance with the STARD reporting checklist (available at https://qims. amegroups.com/article/view/10.21037/qims-21-521/rc).

\section{Methods}

\section{Studied patients}

This study was approved by the Hospital Ethics Committee Review Board (No. 2017-926). The study was conducted in accordance with the Declaration of Helsinki (as revised in 2013). This was a retrospective study. The informed consents were waived in this study. Eight heart transplant recipients enrolled in our hospital between August 2017 and May 2019 underwent CCTA exams prior to heart transplantation. Inclusion criteria were age 30 to 85 years and sinus rhythm. Exclusion criteria included prior reaction to iodinated contrast materials, and impaired renal function (eGFR $<30 \mathrm{~mL} / \mathrm{min} / 1.73 \mathrm{~m}^{2}$ ). All eight patients had been diagnosed with coronary heart disease, previous myocardial infarction, and heart failure. The indication for heart transplantation was heart failure with enlarged left ventricle and ejection fraction $\leq 20 \%$.

\section{CCTA scanning protocols}

All patients in this study routinely underwent non-enhanced scan to measure the calcium score. Thereafter, CCTA was performed. All subjects underwent contrast-enhanced CCTA on the same CT scanner (Discovery CT 750 HD, GE Healthcare, Milwaukee, WI, USA). Acquisition parameters included $64 \times 0.625 \mathrm{~mm}$ detector collimation, $350 \mathrm{~ms}$ gantry rotation time, 170 to $200 \mathrm{~mm}$ field of view, $512 \times 512$ pixel matrix and field of view/pixel matrix pixel size. The energy spectrum scan was set-up such that the bulb voltages were 80 and $140 \mathrm{kVp}$, respectively, and the two tube voltages were switched between 0.3 and $0.5 \mathrm{~s}$. All studies were performed in a cranio-caudal direction with prospective electrocardiographically triggered acquisition during end inspiration. The scan range extended from the level of the carina to just below the dome of the diaphragm. We used a triple phase contrast medium injection protocol which consisted of undiluted contrast agent (Iopamidol, Shanghai Bolaikexin Pharmaceutical Co., China) for 10-12 s followed by a $30 \mathrm{~mL} \mathrm{30 \% :70 \%}$ mixture of contrast medium with an injection time of $8 \mathrm{~s}$; in the third phase, $30-\mathrm{mL}$ saline chaser bolus at flow rates of $5 \mathrm{~mL} / \mathrm{s}$.

All images were reconstructed with a modified iterative reconstruction (ASiR) technique. Previous research demonstrated that ASiR 40-60\% provided the optimum image quality on CCTA (8). Therefore, in this study, we used the ASiR 40\% reconstructions, and uploaded $70 \mathrm{keV}$ images to the post-processing workstation (AW4.6, GE) for analysis in this study (8).

\section{Histopathological analysis}

The histological preparation and analyses were performed 
by experts specialized in cardiovascular pathology. Paraffin sections were obtained in $2-\mathrm{mm}$ and in $3-\mathrm{mm}$ increments (64 and 23 cuts, respectively; 5 stent containing segments were excluded from this study). The thickness of a single histological section was $2 \mathrm{~mm}$. All sections were stained with hematoxylin-eosin and elastic fibers. We used Elastic van Gieson stain specific to elastic fibers.

Each cross-section was classified as vulnerable plaque features if met one or more criteria described below: active inflammation (it refers to the large accumulation of macrophages, $\mathrm{T}$ lymphocytes, and mast cells. There is no specific quantification, but it must be $>15 / \mathrm{mm}^{2}$ or $>15$ per 10 -fold field of view); fibrous cap thickness $<65 \mu \mathrm{m}$ and lipid core accounting for $>40 \%$ of the plaque's total area; endothelial denudation with superficial platelet aggregation or plaque erosion; fissured or injured Plaque; intraplaque hemorrhage. Otherwise "stable" (9).

\section{In vivo CCTA review and match of the histopathological findings}

One observer (X.X1.), who did not take part in the image assessment, performed the co-registration of CCTA and histopathological images. To perfectly match the short axis of CT with the pathological cross-section, we mainly use three methods for co-registration: (I) the distance from a lesion to aortic sinus; (II) coronary vascular bifurcation used as a marker; (III) vascular calcification, stent or bypass position used as another marker. Two experienced radiologists (X.X2. and X.X3.), who did take part in the image assessment, confirmed the co-registration of CCTA images. Special markers and vessel lengths were used to identify target plaque positions and made sure the CCTA trans-axial images were matched to the histological images. Stented and bypassed coronary artery sections were excluded.

\section{Plaque classification}

An experienced radiologist (X.X2.), with 8 years of experience in CCTA imaging, performed the qualitative interpretation of all CCTA cross-sections and assessed the images for the presence and composition of plaque as described below. Another observer (X.X4.), with 11 years of experience in cardiac CT imaging, performed an independent analysis of all sections to assess inter-observer variability. All readings were performed with an adjustable window setting according to the degree of calcification [1,000-1,500 Hounsfield units (HU) window width,
200-750 HU window level].

First, any discernible structure $>1 \mathrm{~mm}^{2}$ that could be assigned to the coronary artery wall, but with a CT attenuation below the contrast-enhanced coronary lumen and above the surrounding connective tissue, was defined as a non-calcified coronary atherosclerotic plaque (10). Plaques meeting this definition and showing any calcified areas were classified as mixed plaque (MP). Any hyperattenuating structure that could be visualized separately from the contrast-enhanced coronary lumen (either it was "embedded" within NCP or its density was above the contrast enhanced lumen) and could be assigned to the coronary artery wall was defined as a calcified atherosclerotic plaque (11). Specifically, we defined the diamond-attenuation-sign (DAS) as a qualitative plaque feature in a MP on CCTA cross-section images by the presence of two features: a visual calcification (in the shape of a diamond) accompanied by an annular-shape lower attenuation plaque tissue surrounding the lumen like a ring. The morphology of DAS including two main morphological features as follows: calcified with large necrotic core-type I, calcified with attenuation-type II.

NRS is a well-known qualitative plaque feature. It was defined as a NCP on CCTA cross-section images by the presence of two features: a central area of low CT attenuation that is apparently in contact with the lumen and a ring-like higher attenuation plaque tissue surrounding this central area (1).

Overall, a total of 271 plaques were characterized according to plaque with or without calcium, according to PCPs, as NCP with NRS (NCP-NRS), and NCP without NRS (NCP-non-NRS), MP with DAS (MP-DAS) and MP without DAS (MP-non-DAS), and pure CP (Figures 1,2).

\section{Statistical analysis}

Continuous variables were expressed as mean \pm standard deviation, and categorical variables were expressed as frequencies or percentages. All statistical tests were performed by using a dedicated statistical software (SPSS 17.0, SPSS Inc., Chicago, IL, USA and MedCalc, version 15, MedCalc Software, Ostend, Belgium). A P value of $<0.05$ was considered statistically significant.

To determine inter-observer variability, an independent reader assessed all CCTA images. The inter-observer agreement was evaluated using Cohen's kappa $(\kappa)$ statistics that were interpreted as follows. A $\kappa$-value greater than 0.80 corresponded to an excellent agreement, a $\kappa$-value of 0.61 

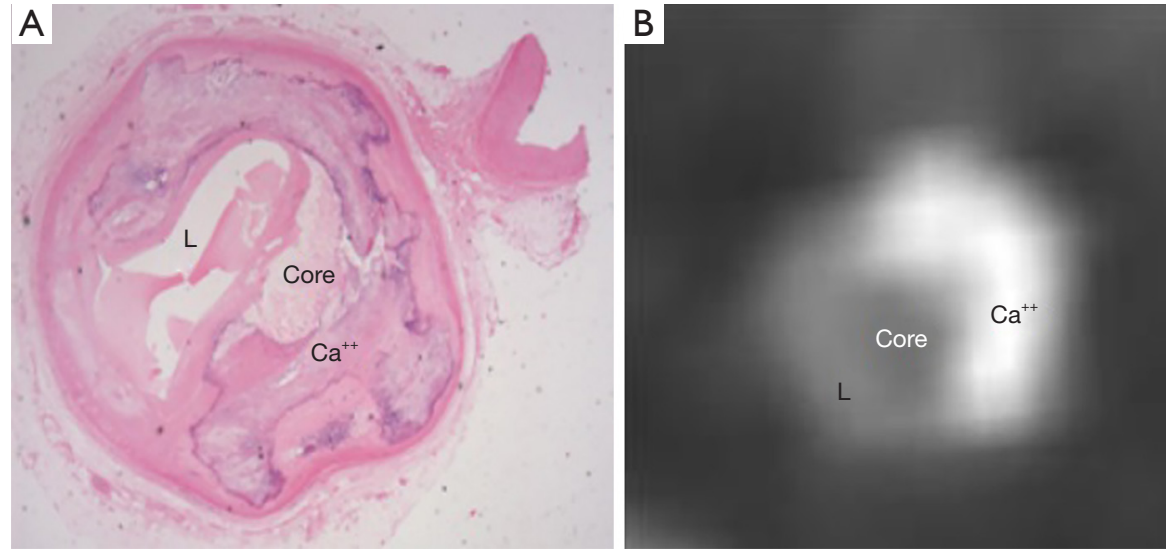

Figure 1 Unstable plaque hide in heavily calcified coronary. Calcification in ruptured coronary plaques. Histologic and CCTA sections from a 65-year-old man who underwent CCTA exams prior to heart transplant surgery. Note large and irregular calcification $\left(\mathrm{Ca}^{++}\right)$with a large lipid core (core), combined with occlusive luminal thrombus. CCTA (right image) showed the presence of two features: a visual obvious calcification (in the shape of a diamond) accompanied by an annular-shape lower attenuation plaque tissue surrounding the lumen like a ring, we defined the DAS as a qualitative plaque feature in a mixed plaque on CCTA cross-section hiding in heavily calcified coronary. CCTA, coronary computed tomography angiography; DAS, diamond-attenuation-sign.

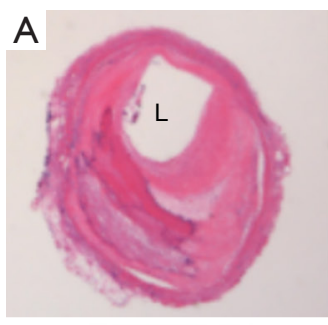

B
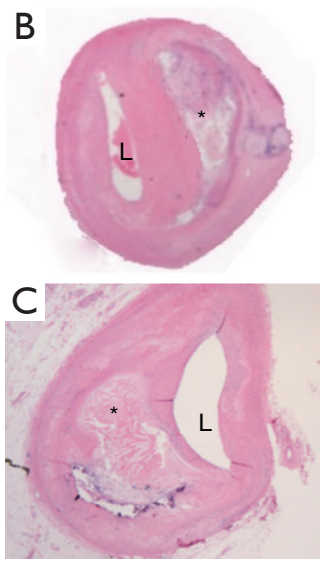
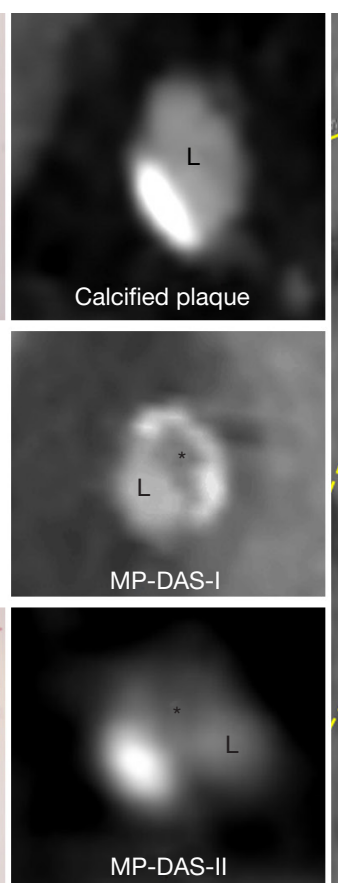
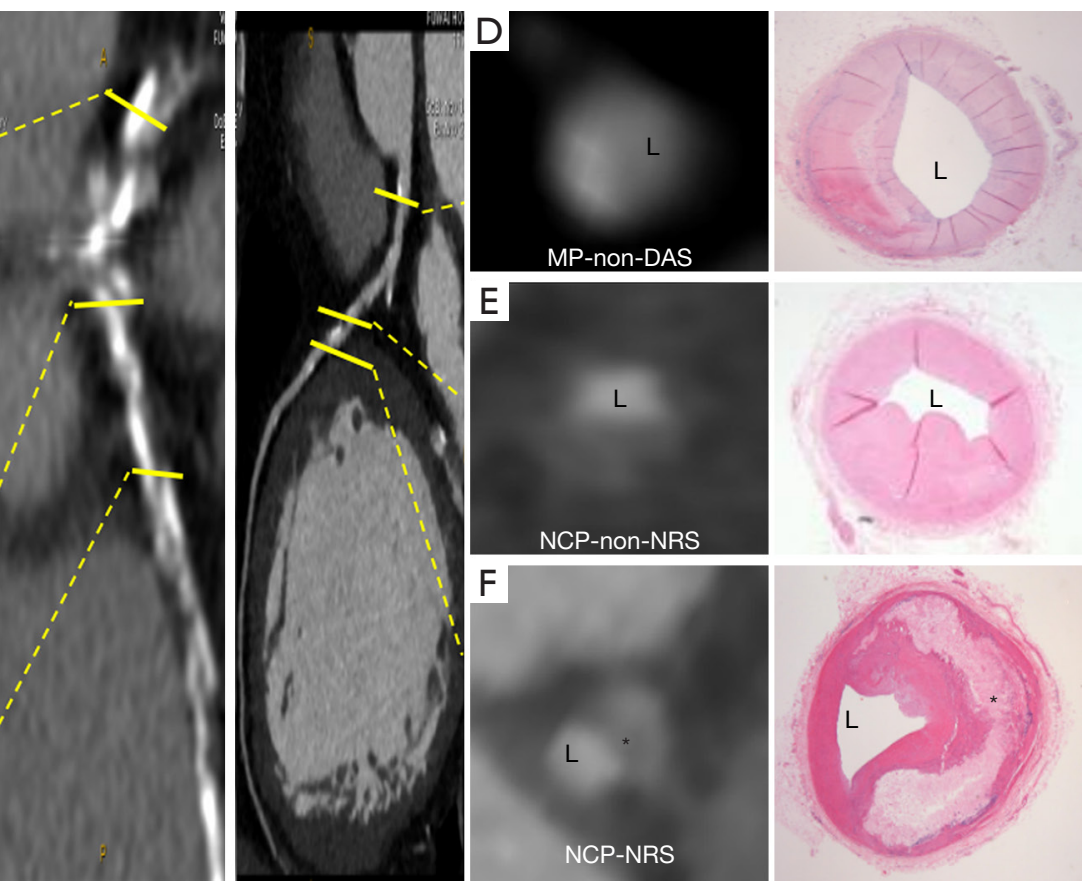

Figure 2 Plaque classifications as assessed by CCTA in vivo. CCTA of an in vivo donor heart with the anatomical position of the plaque cross sections. The plaque-calcified-pattern included calcified (A), MP-DAS-type I (B), MP-DAS-type II (C), MP-non-DAS (D), NCP-NRS (E), NCP-non-NRS (F). The corresponding histology slides: (A) pure calcified; (B) calcified with fibrous cap thickness $<65 \mu \mathrm{m}$ and lipid core accounting for $>40 \%$ of the plaque's total area-type I; (C) calcified with fibrous cap thickness $<65 \mu \mathrm{m}$ and lipid core accounting for $>40 \%$ of the plaque's total area-type II; (D) calcified and fibrous plaque; (E) pathological intimal thickening; (F) late fibroatheroma with fibrous cap thickness $<65 \mu \mathrm{m}$ and lipid core accounting for $>40 \%$ of the plaque's total area. *, necrotic core. L, lumen; MP, mixed plaque; DAS, diamond-attenuation-sign; NCP, non-calcified plaque; NRS, napkin-ring-sign; CCTA, coronary computed tomography angiography. 
Table 1 Patient characteristics

\begin{tabular}{lc}
\hline Characteristics & N (range or \%) \\
\hline Age (years) & $\begin{array}{c}48.5 \pm 11.6 \\
\text { (range, 37-65) }\end{array}$ \\
Male & $8(100 \%)$ \\
Hypertension & $4(50 \%)$ \\
Hyperlipidemia & $5(62.5 \%)$ \\
Diabetes mellitus & $3(37.5 \%)$ \\
Angina pectoris & $8(100 \%)$ \\
Family history of coronary artery disease & $2(25 \%)$ \\
Smoking history & $3(37.5 \%)$ \\
Obesity & $4(50 \%)$ \\
Prior presentation with congestive heart failure & $8(100 \%)$ \\
History of old myocardial infarction & $8(100 \%)$ \\
History of percutaneous intervention & $4(50 \%)$ \\
History of coronary artery bypass graft & $1(12.5 \%)$ \\
\hline
\end{tabular}

to 0.80 corresponded to a good inter-observer agreement, and a $\kappa$-value $<0.61$ corresponded to a poor agreement. We determined the plaque distribution of the PCP categories and the frequency of unstable lesions. To test for statistical significance, the Fisher exact test was used for $2 \times 2$ tables and chi-squared test for tables with more rows or columns. To determine the diagnostic accuracy of CCTA based plaque composition for the detection of unstable lesions, sensitivity, specificity, negative predictive value, and positive predictive value were calculated from $2 \times 2$ contingency tables. We calculated binomial $95 \%$ CI. To further assess the diagnostic capacity of CCTA to detect unstable lesions, the receiver operating characteristic curve (ROC) analysis was used. In the first step, the categories for each classification scheme were sorted separately by their likelihood ratios for unstable lesions. Next, separated logistic regression models for each scheme and area-under-curve (AUC) were calculated. The asymptotic $95 \%$ CI for the AUC were estimated using a non-parametric approach and comparisons in AUC were performed by using a contrast matrix.

\section{Results}

The demographics of the eight heart transplant recipients ( 8 male; mean age, $48.5 \pm 11.6$ years; range, $37-65$ years) who underwent CCTA exams prior to heart transplant surgery are shown in Table 1. Overall, histopathological sections from 27 coronary arteries of eight recipient hearts were investigated. We studied a length of $1,365 \mathrm{~mm}$ in 27 coronary vessels. A total of 358 sections were analyzed and compared both on pathological and CCTA sections. There were 208 stable and 150 unstable lesions confirmed by histopathologic findings. Of the 150 unstable lesions, 86 with active inflammation; 98 with fibrous cap thickness $<65 \mu \mathrm{m}$ and lipid core accounting for $>40 \%$ of the plaque's total area; 36 with endothelial denudation with superficial platelet aggregation or plaque erosion; 22 with fissured or injured Plaque; 14 with intraplaque hemorrhage.

\section{CCTA findings}

At CCTA, no plaque was detected in 87 (24.3\%) sections, NCP in 109 (30.4\%), MP in 137 (38.3\%), and CP in 25 (7.0\%) sections. Furthermore, prevalence of NCP-NRS was $6.4 \%$ (23/358), NCP-non-NRS was $24.0 \%(86 / 358)$, MP-DAS was $18.2 \%(65 / 358)$, MP-non-DAS was $20.1 \%$ (72/358) (Table 2).

\section{Inter-observer variability}

Among the 271 plaques, the inter-observer agreement between the two CCTA readers to classify CCTA sections as no plaque, NCP-NRS, NCP-non-NRS, MP-DAS, MP-nonDAS or Ca-plaque was excellent $(\kappa=0.863)$. Furthermore, the inter-observer agreement to detect DAS and NRS was also excellent ( $\kappa=0.896$ and $\kappa=0.870$, respectively).

\section{Comparison of CCTA plaque type to bistopathological findings}

Features of plaque vulnerability on histopathology were correlated with findings of NRS in NCP or DAS in MP, based upon CCTA. Overall, the percentage of unstable atherosclerotic lesions among different CT plaque types were as follows: $86.9 \%$ of NCP-NRS (20/23), $33.7 \%$ of NCP-non-NRS (29/86), 90.8\% of MP-DAS (59/65), 52.7\% of MP-non-DAS (38/72), and $16.0 \%$ of Ca-plaque (4/25), with histopathological findings serving as the reference standard (Figure 3, all $\mathrm{P}<0.001$ ).

In addition, most commonly on DAS plaques, the areas of the necrotic cores were large and the proportion within the total plaque area of MP-DAS were higher than in MP-nonDAS (47.8\% vs. $21.4 \%, \mathrm{P}<0.001)$, similar to NRS (47.8\% vs. $45.4 \%, \mathrm{P}=0.01)$. This finding suggests that the presence 
Table 2 Plaque-calcification-pattern based plaque classification for the differentiation of stable and unstable lesions confirmed by histopathologic findings

\begin{tabular}{lccc}
\hline Plaque-calcification-pattern scheme & Stable & Unstable & In total \\
\hline No plaque, n (\%) & $87(41.8)$ & $0(0)$ & $47(24.3)$ \\
CP, n (\%) & $21(10.1)$ & & $25(7.0)$ \\
NCP, n (\%) & & $20(13.3)$ & $23(6.4)$ \\
With NRS (NCP-NRS) & $3(1.4)$ & $29(19.3)$ & $86(24.0)$ \\
Without NRS (NCP-non-NRS) & $57(27.4)$ & & $65(18.2)$ \\
MP, n (\%) & $6(2.9)$ & $38(25.3)$ & $72(20.1)$ \\
With DAS (MP-DAS) & $34(16.4)$ & $150(100.0)$ & $358(100.0)$ \\
Without DAS (MP-non-DAS) & $208(100.0)$ & \\
In total
\end{tabular}

Note: $\mathrm{P}<0.0001$. CP, calcified plaque; NCP, non-calcified plaque; NRS, napkin-ring-sign; MP, mixed plaque; DAS, diamond-attenuation-sign.

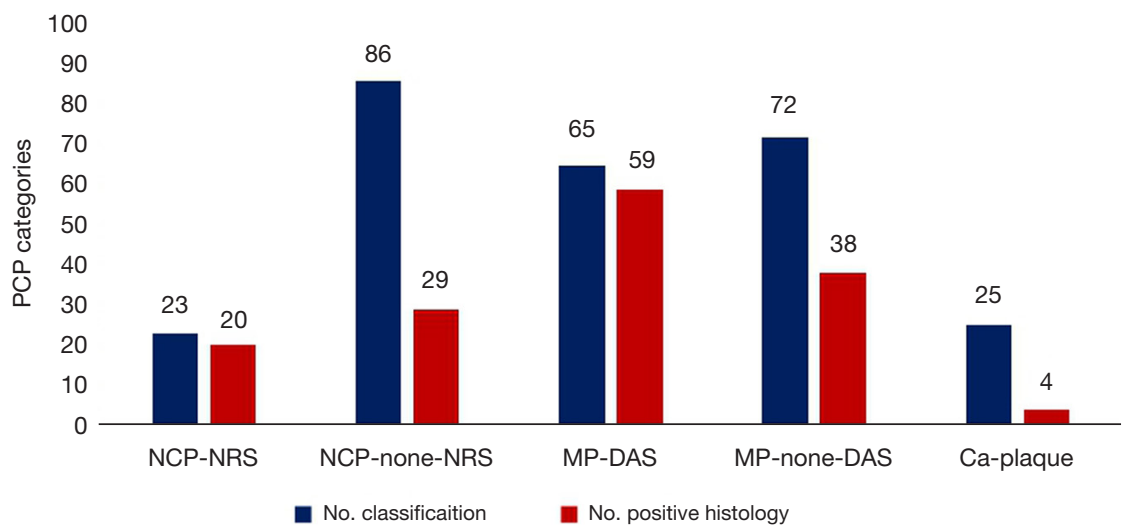

Figure 3 Number of CT plaque types among plaque-calcification-based pattern in vivo. The blue bars represent number of CT showed plaques base on PCP model. The red bars represent unstable atherosclerotic lesions of each type. There were statistical significances between them (all $\mathrm{P}<0.001$ ). NCP-NRS indicates non-calcified plaque with napkin-ring-sign; NCP-none-NRS indicates non-calcified plaque without napkin-ring-sign; MP-DAS indicates mixed plaque with diamond-attenuation-sign; MP-none-DAS indicates mixed plaque without diamond-attenuation-sign; Ca-plaque indicates calcified plaque. PCP, plaque-calcification-pattern; NCP, non-calcified plaque; NRS, napkin-ring-sign; MP, mixed plaque; Ca-plaque, calcified plaque; DAS, diamond-attenuation sign.

of NRS or DAS was independently associated with unstable plaque features on histopathology. However, DAS plaques were detected more frequently by CCTA exams than the NRS type (39.3\% vs. $13.3 \%$, respectively, $\mathrm{P}=0.001)$ (Table 3).

\section{Diagnostic accuracy}

DAS and NRS both showed a high specificity $(97.1 \%$ vs.
98.6\%) and positive predictive value (90.8\% vs. $87.0 \%)$ to identify unstable plaque features on histopathology. ROC analysis revealed that the AUC of the PCP to identify unstable lesions was 0.883 (95\% CI: $0.845-0.914)$. The results of DAS (AUC, 0.756 ; 95\% CI: 0.717-0.791) as a CCTA feature to identify coronary unstable lesions were better than that of NRS (AUC, 0.558; 95\% CI: 0.514-0.600) $(\mathrm{P}<0.001$, Figures 4,5). 
Table 3 Different qualitative plaque scheme for CCTA in vivo based on PCP model classified by histology with 95\% CI

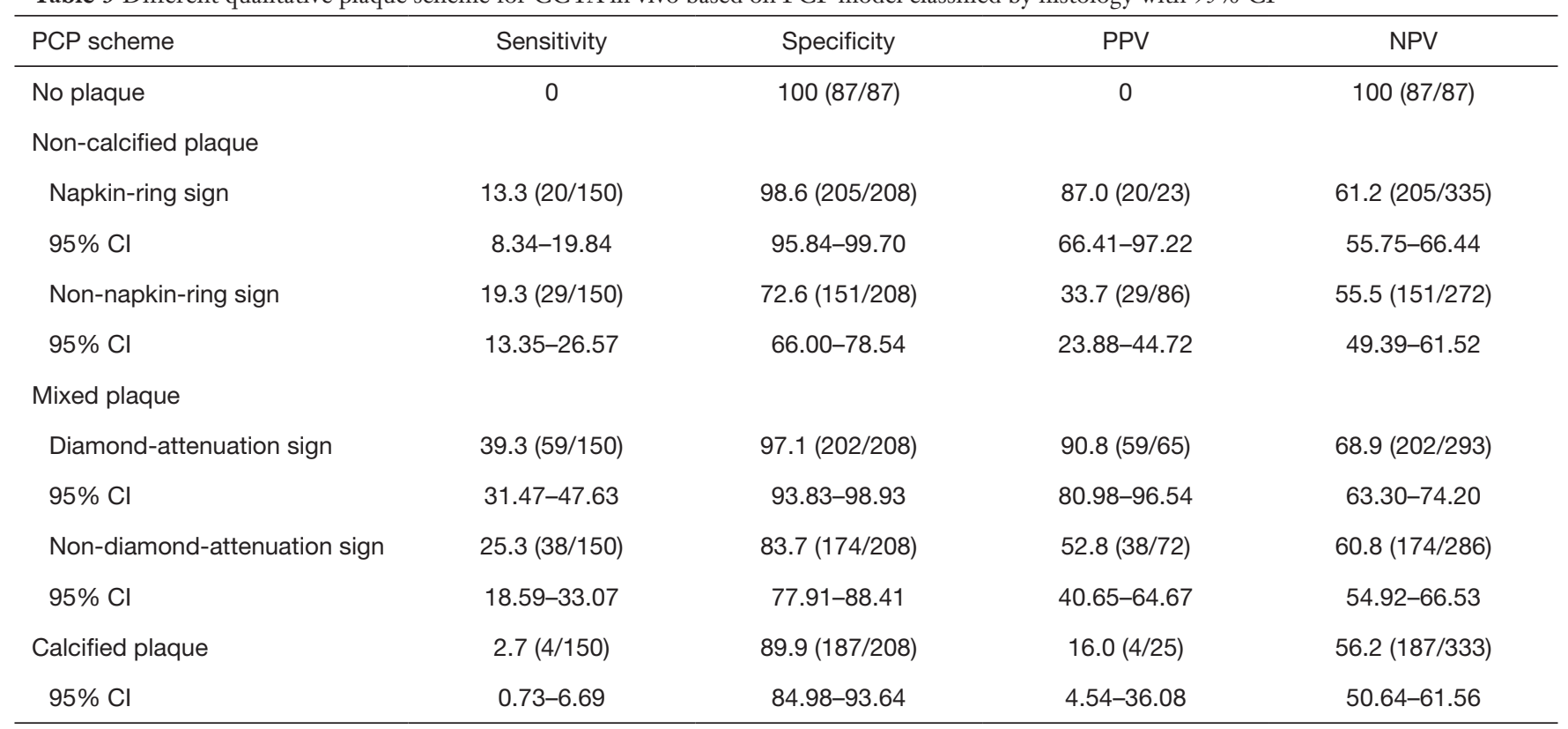

Values are (\%). CCTA, coronary computed tomography angiography; PCP, plaque-calcification-pattern; PPV, positive predictive value; NPV, negative predictive value.

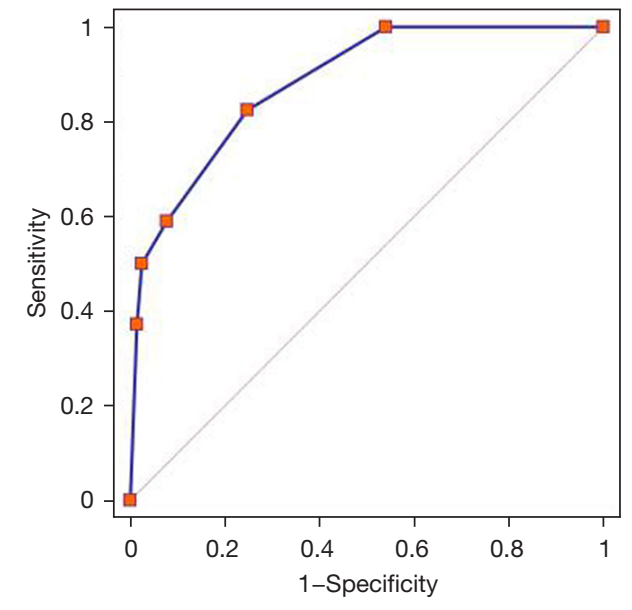

Figure 4 ROC curves of the PCP scheme. The area under the ROC of the PCP scheme (the blue line) to identify unstable lesions was 0.883 (95\% CI: $0.845-0.914, \mathrm{P}<0.0001$ ). ROC, receiveroperating characteristic; PCP, plaque-calcification-pattern.

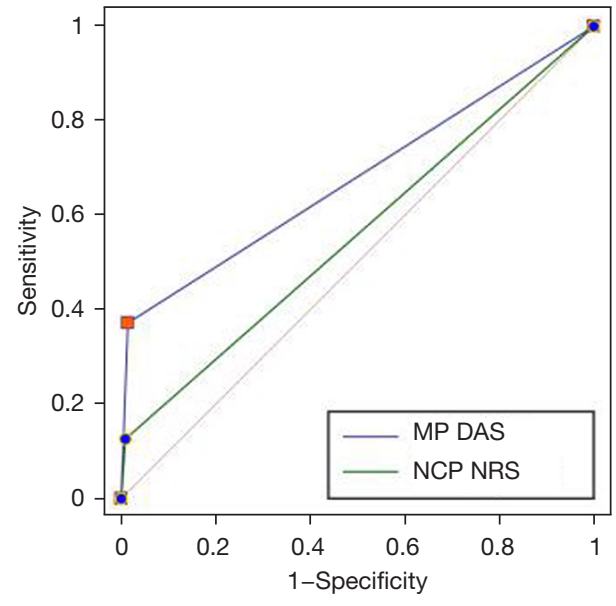

Figure 5 ROC curves to compare DAS versus NRS in our PCP scheme. The area under the ROC curve was 0.756 for DAS (the blue line) and 0.558 for NRS (green line), $\mathrm{P}<0.001$. MP, mixed plaque; DAS, diamond-attenuation sign; NCP, noncalcified plaque; NRS, napkin-ring-sign; ROC, receiver-operating characteristic (95\% CI: $0.717-0.791$ vs. 0.514-0.600); PCP, plaquecalcification-pattern. 


\section{Discussion}

Calcification is commonly found in atherosclerosis and the presence and extent of calcification is associated with a poor prognosis (12). Moreover, ongoing efforts to prevent acute coronary events assume that these events will happen at the level of individual plaques (13) in patients unstable to plaque rupture. However, the impact of the calcification formation within a specific lesion on the differentiation of stable and unstable atherosclerotic lesions remains controversial (14-16). This study adds to the existing knowledge by demonstrating that the assessment of PCPs improves the diagnostic accuracy of CCTA to identify unstable atherosclerotic lesions in vivo. Moreover, we found that the newly defined DAS in CCTA cross-sectional images showed a high specificity and positive predictive value for the presence of unstable lesions.

Maurovich-Horvat et al. proposed that the NRS is a qualitative CCTA plaque feature to define unstable plaques (7). It is thought to represent a CT specific feature of NCPs with large necrotic cores. However, a more detailed analysis of different plaque attenuation patterns may provide improved diagnostic performance and better evaluation of unstable coronary plaques $(7,17)$. Our findings were consistent with the previously published studies that proved MP, rather than NCP, had a higher prevalence in clinical CCTA practice (18). Our data also suggested that plaques with NRS indicated unstable lesions that are consistent with the studies by Hoffman et al. (19), although the majority of analytical discrepancies between the two CCTA readers resulted from an inadequate spatial resolution of CCTA images to differentiate the NRS from non-NRS.

In this study, heart transplant recipients with coronary heart disease underwent CCTA exams prior to heart transplant surgery (20). The atherosclerotic lesions in large parts of the specimens were MPs. The CCTA image quality in this study was representative of real-world routine exams with image quality adequate enough to allow good inter-observer agreement to identify either NCPNRS or MP-DAS. Our data suggests that certain qualities of MP like presence of obvious calcium and lipid-core tissue are associated with unstable atherosclerotic lesions. Moreover, there was satisfactory differentiation between CP components and lipid-rich plaque, which is consistent with earlier findings (21).

Our data also demonstrates that the absence of plaque at CCTA largely excludes the presence of unstable atherosclerotic lesions, which is consistent with prior ex vivo and clinical studies demonstrating the rarity of cardiovascular events in patients free of coronary atherosclerosis by CCTA $(22,23)$. Unexpectedly, we found four unstable plaques on histopathology that had appeared as purely calcified lesions on CCTA. This is likely a result of blooming artifacts caused by calcification so that a small area of lipid component undetected on CCTA images (24).

Overall, the influence of calcification on plaque stability remains a topic of interest. On a clinical level, recent studies showed that coronary artery calcium among individuals aged between 32 and 46 years was associated with increased risk of fatal and nonfatal coronary heart disease during 12.5 years of follow-up (25), which adds to the known predictive power of coronary artery calcium. On a more basic level, studies showed that calcification did not increase fibrous cap stress in ruptured or stable human coronary atherosclerotic lesions (26).

Lesions presenting as DAS on CCTA often have a large necrotic lipid-rich core. The distinct features of DAS on CCTA, according to our observations, require the presence of a MP with an especially high proportion of lipid admixture, as confirmed by our histopathological findings.

Many prior studies have shown that plaques demonstrating acute rupture typically had mild or moderate calcification associated with extensive and diffuse coronary atherosclerosis and accelerated growth $(27,28)$. The high association of the DAS plaque-type with features of vulnerability on CCTA may be attributable to calcification as an osteogenic process in response to inflammatory reactions, which would reflect an active state associated with extensive plaque volume and accelerated progression. As a result, DAS likely consists of a soft lipid component coexisting with calcification and increasing plaque vulnerability.

The results of this study should be interpreted within the context of the study design and consequent limitations. Prospective studies and clinical investigations in vivo are necessary to assess the generalizability and reproducibility of our findings. First, all CT studies were acquired in heart transplant recipients who had coronary heart disease in vivo, so the patient group was necessarily small and the atherosclerotic lesions in large parts of the specimens were calcified or mixed; and only one sex in the analysis; this limits the generalizability of our results. Second, due to the limited spatial resolution of CCTA, smaller vessels were excluded from assessment in our investigation. There are limitations in plaque characterization by CT 
incurred through the "masking" of NCP components by calcification, which appears larger in CT than its actual size due to blooming artifacts. On the one hand, CT histogram analysis of the plaques could provide greater confidence in the appropriate categorising of this non-calcified lipid-core plaque phenotype (29). On the other hand, calcification specific staining such as Alizarin red, Von Kossa or Picosirus Red was not used in our study. Especially, it was more advantageous for a small amount of calcification or micro-calcification and could be quantified in hitology. In addition, some small amount of calcification or microcalcification would be missed in CCTA because of luminal contrast enhancement and limited resolution. The area and arc of calcification around the plaque would differ between histology and CCTA. This was why the calcification plaques with DAS appeared to be 'nodule', while the specimens in histology looked like sheets of calcification that extended around 120 degrees of the arc per se. Third, many unstable plaque features found on histopathology could not be reproduced when using CCTA. Validation of the diamond attenuation sign in a large CT registry is warranted to ascertain the true prevalence of this plaque phenotype. Thus further clinical investigations in vivo would be required to assess the reproducibility of our findings and strengthen the observations in this study.

In conclusion, the qualitative assessment of the PCP in patients with coronary atherosclerosis improved diagnostic accuracy of CCTA to identify unstable lesions hiding in heavily calcified coronary arteries, including our newly defined "diamond-attenuation-sign (DAS)", which are more frequently seen on CCTA images.

\section{Acknowledgments}

Abstract of this manuscript has been presented in The 13th Congress of Asian Society of Cardiovascular Imaging.

Funding: This work was supported by the Ministry of Science and Technology of China, National key research and development project (No. 2016YFC1300400), the National Natural Science Foundation of China (No. 82102036), Fundamental Research Funds for the Central Universities (No. 3332018063) and The Doctoral Research Startup Fund 2021 of GuiZhou Medical University (No. GYFYBSKY-2021-49), The cultivate project 2021 for National Natural Science Foundation of China, Affiliated Hospital of Guizhou Medical University (No. GYFYNSFC-2021-32).

\section{Footnote}

Reporting Checklist: The authors have completed the STARD reporting checklist. Available at https://qims.amegroups. com/article/view/10.21037/qims-21-521/rc

Conflicts of Interest: All authors have completed the ICMJE uniform disclosure form (available at https://qims. amegroups.com/article/view/10.21037/qims-21-521/coif). The authors have no conflicts of interest to declare.

Ethical Statement: The authors are accountable for all aspects of the work in ensuring that questions related to the accuracy or integrity of any part of the work are appropriately investigated and resolved. This study was approved by the Hospital Ethics Committee Review Board (No. 2017-926). The study was conducted in accordance with the Declaration of Helsinki (as revised in 2013). This was a retrospective study. The informed consents were waived in this study.

Open Access Statement: This is an Open Access article distributed in accordance with the Creative Commons Attribution-NonCommercial-NoDerivs 4.0 International License (CC BY-NC-ND 4.0), which permits the noncommercial replication and distribution of the article with the strict proviso that no changes or edits are made and the original work is properly cited (including links to both the formal publication through the relevant DOI and the license). See: https://creativecommons.org/licenses/by-nc-nd/4.0/.

\section{References}

1. Maurovich-Horvat P, Ferencik M, Voros S, Merkely B, Hoffmann U. Comprehensive plaque assessment by coronary CT angiography. Nat Rev Cardiol 2014;11:390-402.

2. Malik S, Zhao Y, Budoff M, Nasir K, Blumenthal RS, Bertoni AG, Wong ND. Coronary artery calcium score for long-term risk classification in individuals with type 2 diabetes and metabolic syndrome from the multi-ethnic study of atherosclerosis. JAMA Cardiol 2017;2:1332-40.

3. Ianiro G, Cammarota G, Gasbarrini A. Letter by Ianiro et al regarding article, "Effect of long-term metformin and lifestyle in the diabetes prevention program and its outcome study on coronary artery calcium". Circulation 2018;137:213-4. 
4. Higashi M. Noninvasive assessment of coronary plaque using multidetector row computed tomography: does MDCT accurately estimate plaque vulnerability? (Con). Circ J 2011;75:1522-8.

5. Hoffmann U, Butler J. Noninvasive detection of coronary atherosclerotic plaque by multidetector row computed tomography. Int J Obes (Lond) 2005;29 Suppl 2:S46-53.

6. Becker CR, Knez A, Ohnesorge B, Schoepf UJ, Reiser MF. Imaging of noncalcified coronary plaques using helical CT with retrospective ECG gating. AJR Am J Roentgenol 2000;175:423-4.

7. Maurovich-Horvat P, Schlett CL, Alkadhi H, Nakano M, Otsuka F, Stolzmann P, Scheffel H, Ferencik M, Kriegel MF, Seifarth H, Virmani R, Hoffmann U. The napkinring sign indicates advanced atherosclerotic lesions in coronary CT angiography. JACC Cardiovasc Imaging 2012;5:1243-52.

8. Fuchs TA, Stehli J, Fiechter M, Dougoud S, Gebhard C, Ghadri JR, Husmann L, Gaemperli O, Kaufmann PA. First experience with monochromatic coronary computed tomography angiography from a 64-slice CT scanner with Gemstone Spectral Imaging (GSI). J Cardiovasc Comput Tomogr 2013;7:25-31.

9. Naghavi M, Libby P, Falk E, Casscells SW, Litovsky S, Rumberger J, et al. From vulnerable plaque to vulnerable patient: a call for new definitions and risk assessment strategies: Part I. Circulation 2003;108:1664-72.

10. Achenbach S, Moselewski F, Ropers D, Ferencik M, Hoffmann U, MacNeill B, Pohle K, Baum U, Anders K, Jang IK, Daniel WG, Brady TJ. Detection of calcified and noncalcified coronary atherosclerotic plaque by contrast-enhanced, submillimeter multidetector spiral computed tomography: a segment-based comparison with intravascular ultrasound. Circulation 2004;109:14-7.

11. Maurovich-Horvat P, Hoffmann U, Vorpahl M, Nakano M, Virmani R, Alkadhi H. The napkin-ring sign: CT signature of high-risk coronary plaques? JACC Cardiovasc Imaging 2010;3:440-4.

12. Taylor AJ, Bindeman J, Feuerstein I, Cao F, Brazaitis M, O'Malley PG. Coronary calcium independently predicts incident premature coronary heart disease over measured cardiovascular risk factors: mean three-year outcomes in the Prospective Army Coronary Calcium (PACC) project. J Am Coll Cardiol 2005;46:807-14.

13. Greenland P, LaBree L, Azen SP, Doherty TM, Detrano RC. Coronary artery calcium score combined with Framingham score for risk prediction in asymptomatic individuals. JAMA 2004;291:210-5.
14. Mannelli L, MacDonald L, Mancini M, Ferguson M, Shuman WP, Ragucci M, Monti S, Xu D, Yuan C, Mitsumori LM. Dual energy computed tomography quantification of carotid plaques calcification: comparison between monochromatic and polychromatic energies with pathology correlation. Eur Radiol 2015;25:1238-46.

15. Huang H, Virmani R, Younis H, Burke AP, Kamm RD, Lee RT. The impact of calcification on the biomechanical stability of atherosclerotic plaques. Circulation 2001;103:1051-6.

16. Dwivedi G, Liu Y, Tewari S, Inacio J, Pelletier-Galarneau $\mathrm{M}$, Chow BJ. Incremental prognostic value of quantified vulnerable plaque by cardiac computed tomography: a pilot study. J Thorac Imaging 2016;31:373-9.

17. Seifarth H, Schlett CL, Nakano M, Otsuka F, Károlyi M, Liew G, Maurovich-Horvat P, Alkadhi H, Virmani $\mathrm{R}$, Hoffmann U. Histopathological correlates of the napkin-ring sign plaque in coronary CT angiography. Atherosclerosis 2012;224:90-6.

18. Otsuka K, Fukuda S, Tanaka A, Nakanishi K, Taguchi H, Yoshikawa J, Shimada K, Yoshiyama M. Napkin-ring sign on coronary CT angiography for the prediction of acute coronary syndrome. JACC Cardiovasc Imaging 2013;6:448-57.

19. Hoffmann U, Moselewski F, Nieman K, Jang IK, Ferencik M, Rahman AM, Cury RC, Abbara S, Joneidi-Jafari H, Achenbach S, Brady TJ. Noninvasive assessment of plaque morphology and composition in culprit and stable lesions in acute coronary syndrome and stable lesions in stable angina by multidetector computed tomography. J Am Coll Cardiol 2006;47:1655-62.

20. Erthal F, Premaratne M, Yam Y, Chen L, Lamba J, Keenan M, Haddad T, Pharasi K, Anand S, Beanlands RS, Burwash IG, Dwivedi G, Ruddy TD, Chow BJW. Appropriate use criteria for cardiac computed tomography: does computed tomography have incremental value in all appropriate use criteria categories? J Thorac Imaging 2018;33:132-7.

21. Bastarrika G, De Cecco CN, Arraiza M, Ubilla M, Mastrobuoni S, Pueyo JC, Rábago G. Dual-source CT coronary imaging in heart transplant recipients: image quality and optimal reconstruction interval. Eur Radiol 2008;18:1791-9.

22. Matsumoto N, Sato Y, Yoda S, Nakano Y, Kunimasa T, Matsuo S, Komatsu S, Saito S, Hirayama A. Prognostic value of non-obstructive CT low-dense coronary artery plaques detected by multislice computed tomography. Circ J 2007;71:1898-903.

23. Nance JW Jr, Schlett CL, Schoepf UJ, Oberoi S, Leisy 
HB, Barraza JM Jr, Headden GF, Nikolaou K, Bamberg F. Incremental prognostic value of different components of coronary atherosclerotic plaque at cardiac CT angiography beyond coronary calcification in patients with acute chest pain. Radiology 2012;264:679-90.

24. Sarwar A, Rieber J, Mooyaart EA, Seneviratne SK, Houser SL, Bamberg F, Raffel OC, Gupta R, Kalra MK, Pien H, Lee H, Brady TJ, Hoffmann U. Calcified plaque: measurement of area at thin-section flat-panel CT and 64-section multidetector CT and comparison with histopathologic findings. Radiology 2008;249:301-6.

25. Carr JJ, Jacobs DR Jr, Terry JG, Shay CM, Sidney S, Liu K, Schreiner PJ, Lewis CE, Shikany JM, Reis JP, Goff DC Jr. Association of coronary artery calcium in adults aged 32 to 46 years with incident coronary heart disease and death. JAMA Cardiol 2017;2:391-9.

26. Richardson PD, Davies MJ, Born GV. Influence of plaque configuration and stress distribution on fissuring of

Cite this article as: Yin WH, Wang HY, Sun Y, Li XN, Lu ZF, An YQ, Hou ZH, Zhang J, Ren XS, Schoepf UJ, Zhang $\mathrm{Y}, \mathrm{Lu} \mathrm{B}$. Unstable plaques hide in heavily calcified coronary arteries. Quant Imaging Med Surg 2022;12(5):2744-2754. doi: 10.21037/qims-21-521 coronary atherosclerotic plaques. Lancet 1989;2:941-4.

27. Maldonado N, Kelly-Arnold A, Vengrenyuk Y, Laudier D, Fallon JT, Virmani R, Cardoso L, Weinbaum S. A mechanistic analysis of the role of microcalcifications in atherosclerotic plaque stability: potential implications for plaque rupture. Am J Physiol Heart Circ Physiol 2012;303:H619-28.

28. Mauriello A, Servadei F, Zoccai GB, Giacobbi E, Anemona L, Bonanno E, Casella S. Coronary calcification identifies the vulnerable patient rather than the vulnerable Plaque. Atherosclerosis 2013;229:124-9.

29. Schlett CL, Maurovich-Horvat P, Ferencik M, Alkadhi H, Stolzmann P, Scheffel H, Seifarth H, Nakano M, Do S, Vorpahl M, Kauczor HU, Bamberg F, Tearney GJ, Virmani R, Hoffmann U. Histogram analysis of lipid-core plaques in coronary computed tomographic angiography: ex vivo validation against histology. Invest Radiol 2013;48:646-53. 